\title{
The Chronic Care for Aboriginal People program in NSW
}

\author{
Raylene Gordon $^{\mathrm{A}, \mathrm{B}}$ and Natalie Richards ${ }^{\mathrm{A}}$ \\ A Primary Health, Community Partnerships and Chronic Disease \\ Management Branch, NSW Ministry of Health \\ ${ }^{\mathrm{B}}$ Corresponding author.Email: rgord@doh.health.nsw.gov.au
}

\begin{abstract}
Aboriginal people living in NSW continue to experience greater health risks, poorer health and shorter life expectancies than nonAboriginal Australians. The NSW Health-funded program, Chronic Care for Aboriginal People, was established from existing initiatives to rethink the way chronic care services were delivered to Aboriginal people in NSW. Refocusing and building on existing projects led to NSW Health providing an evidence base of what was working in Aboriginal communities. A model of care for Aboriginal people with chronic disease has been developed. Recommendations from two evaluations have allowed further improvement for the delivery of chronic care services for Aboriginal people. The Local Health District Service Agreements include relevant indicators and strategic priorities relating to the chronic care program.
\end{abstract}

Aboriginal people are generally less healthy than other Australians, die at much younger ages and suffer more chronic conditions and a lower quality of life. ${ }^{1}$ In New South Wales (NSW), Aboriginal people experience a disproportionately high burden of chronic conditions, including cardiovascular disease, kidney disease, chronic respiratory disease and diabetes, which significantly contributes to their greater morbidity and premature mortality compared to non-Aboriginal Australians. ${ }^{2,3}$ For example, hospitalisation rates for Aboriginal people compared to non-Aboriginal people in NSW are 70\% higher for cardiovascular diseases, $200 \%$ higher for diabetes and 100\% higher for chronic respiratory disease. ${ }^{2}$

The challenges of managing chronic disease are compounded by the complexity of the conditions and the need of patients to access multiple service providers in different sectors over an extended period. ${ }^{2}$ For Aboriginal people, chronic disease is associated with cultural, economic, social and historical issues which impact on service access and uptake. Because the current health-care system often does not adequately meet the needs of Aboriginal people in culturally appropriate ways, NSW Health set out to develop a model of care for addressing chronic diseases in Aboriginal communities. This initiative would then provide an evidence base for the effective management and treatment of chronic diseases in Aboriginal people. This article provides an overview of the program of Chronic Care for Aboriginal People and how it evolved from the Walgan Tilly Project and other programs.

\section{Chronic Care for Aboriginal People}

In 2008, the Chronic Care for Aboriginal People program evolved from a number of existing initiatives such as the Aboriginal Vascular Health Program (2000) and the Aboriginal Chronic Conditions Area Health Service Standards $(2005)^{4}$ to address the disparities of care particularly for heart disease, respiratory disease, diabetes and renal disease. The goals of the program were to: provide methods to improve access to chronic disease services for Aboriginal people; build working relationships between Aboriginal and chronic disease services in NSW; and identify and share best practice in meeting the needs of Aboriginal people with chronic disease.

\section{Walgan Tilly Redesign Project}

Clinical redesign is an improvement method that focuses on the patient journey in accessing emergency and elective care. ${ }^{5}$ The first Aboriginal specific redesign process, the Walgan Tilly Redesign Project, was established by NSW Health with clinicians, managers, patients and carers to increase the access of Aboriginal people to health services in public hospitals in NSW. The Project started in October 2007 and involved extensive consultation with staff, patients and carers across NSW. The findings from these consultations, as well as expert advice and reviews of the literature helped identify solutions to closing the gap in health outcomes between Aboriginal and non-Aboriginal people. These solutions included a model of care for Aboriginal people, greater emphasis on cultural awareness and sensitivity of services, and the integration of Aboriginal health and chronic disease into mainstream services. ${ }^{6}$ Each of the former Area Health Services, identified local solutions to their local issues. These solutions were implemented through all former Area Health Services and Justice Health over a 2-year period from July 2008 to June 2010. 
As a result of the Walgan Tilly Redesign Project, the Chronic Care for Aboriginal People program specifically addresses four major diseases: heart disease, diabetes, chronic lung disease and kidney disease. Implementation was coordinated by the NSW Ministry of Health in collaboration with Local Health Districts and other stakeholders including local Aboriginal Medical Services and General Practice NSW.

\section{Evaluation: Walgan Tilly Redesign Project}

To assist in informing future planning and service development, the Walgan Tilly Redesign Project was evaluated over the period December 2010 to April 2011 to determine the impact of the Project on Aboriginal health inequalities, the effectiveness of the identified Walgan Tilly solutions and to assess the appropriateness of the approaches that were used.

The evaluation was conducted through extensive consultation (surveys and interviews) with stakeholders involved in the implementation of the project at both a state and local level. The evaluation confirmed the significant achievements and changes resulting from the Walgan Tilly Redesign Project. One of the findings was the importance of having a statewide focus on Aboriginal chronic disease. A number of factors that contributed to the success of the Walgan Tilly Project were identified and have broader applicability for the development and implementation of future Aboriginal health programs. They include:

- A statewide focus on Aboriginal chronic care and mandated key performance indicators

- Senior Executive and other Local Health District support

- Clinical redesign methodology

- Committed people working together effectively

- Central roles for Aboriginal Health Workers

- Identified lead coordination position

- Aboriginal community engagement

- Structured reporting timeframes

- Innovation and opportunity.

\section{Recommendations of the evaluation}

The evaluation identified factors that would have strengthened the statewide project, such as better governance and accountability, communication and consultation, consistency and timeliness of providing information and Aboriginal health lead roles. The evaluation also identified ways to continue to improve the delivery of chronic care services for Aboriginal people including engaging local communities and allowing time for working relationships to develop.

\section{A model of care for Aboriginal people}

The development of a model of care for Aboriginal people with, or at risk of developing, a chronic disease was one of the outcomes from the Walgan Tilly Redesign Project findings. Eight fundamental elements were identified as being essential to a framework for managing chronic disease in Aboriginal communities: identification, trust, screening and assessment, clinical indicators, treatment, education, referral and follow-up (Figure 1). ${ }^{6,7}$

This model complements existing structures that support improving health outcomes for Aboriginal people with, or at risk of developing, a chronic disease. The model based on statewide best practice:

- identifies gaps and opportunities at a local and state level to maximise existing resources

- provides an opportunity for new initiatives to address chronic diseases in Aboriginal communities

- provides a platform for partnerships with service providers within existing health and social networks.

The most significant difference between this model and other models of care is the importance placed on trust and mutual respect. Aboriginal people have suffered significant losses including family, land, identity and language. As a result, many Aboriginal people do not trust mainstream health services. The model of care provides an approach that is respectful of how difficult these factors are to overcome.

\section{Hour Follow-up program}

A recommendation arising from the Walgan Tilly Project was that all Aboriginal people aged 15 years and older, who were admitted to an acute care facility, and who had a chronic disease (heart disease, diabetes, chronic lung disease and kidney disease), should be followed up within 2 working days of discharge. 48 Hour Follow-up program has been implemented across the state in over 60 hospitals. The program's target is for $90 \%$ of eligible patients identified to be followed up within 2 working days.

\section{Evaluation of the 48 Hour Follow-up program}

A preliminary evaluation was conducted after 1 year of the program's implementation. The evaluation was conducted through patient and staff surveys, an analysis of data provided by the Local Health Districts and inpatient data from the health information exchange (HIE). The data analysis reviewed how well the $90 \%$ target was being met, how well hospitals were identifying eligible patients and the effect of re-admissions for people being followed up. A literature review on similar follow-up interventions was also undertaken.

\section{Recommendations of the evaluation}

The evaluation made a series of recommendations including:

- Reviewing key performance indicators and data sources and assessing the ability of Local Health Districts to locate eligible patients within their facilities to ensure all eligible patients are identified and offered follow-up.

- Examining hospital data for inconsistencies between data sources.

- Providing additional resources for clinical support and intervention as well as the expanded use of home visits, 


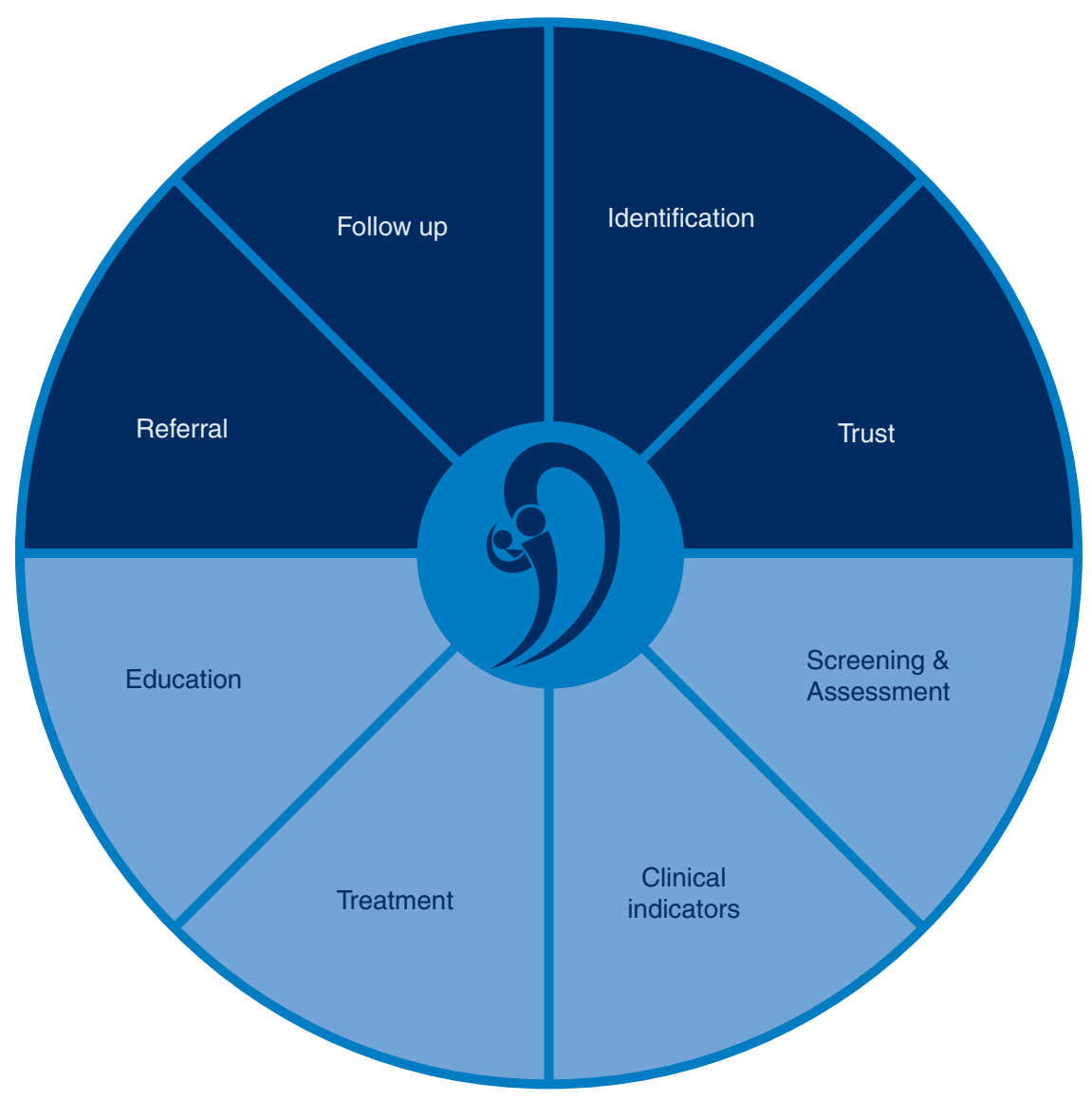

Figure 1. The model of care developed for the Chronic Care for Aboriginal People program.

Source: NSW Health Chronic care for Aboriginal people. September 2010. ${ }^{7}$

and the promotion of the service to eligible patients while they are in hospital.

- Expanding the program to specifically seek information about new problems and personal support available after discharge and investigating the benefits of home-visit follow-up.

\section{Next steps}

All Local Health Districts will adopt the new model of care for enhancement to the 48 Hour Follow-up program, which includes these components:

- dedicated positions for the 48 Hour Follow-up program

- having a central point of contact

- clinical supervision and support

- improved identification of eligible patients for follow-up

- increased capacity for home visiting in addition to a 48 hour follow-up phone call.

The evaluation of the enhanced 48 Hour Follow-up program is being funded through the National Partnership Agreement on Closing the Gap in Indigenous Health Outcomes ${ }^{8}$ and will be undertaken jointly between the Centre for Aboriginal Health and the University of Newcastle. The proposed intervention includes strategies designed to assist the health-care system to be more sensitive to the cultural, health and socio-economic needs of Aboriginal patients with chronic disease. Guided by an advisory group, the intervention will consist of the following strategies:

- recommendations for clinicians involved in the patient's care for improving treatment adherence

- care delivered by the Chronic Care Worker while the patient is still in hospital

- care delivered post discharge by the Aboriginal Chronic Care Worker

- feedback to the health-care system.

Currently, the Local Health District Service Agreements include relevant indicators and strategic priorities relating to the chronic care program.

\section{Connecting Care Program}

The Chronic Care for Aboriginal People program is working closely with the Connecting Care Program which was introduced to former Area Health Services in 2009. The Connecting Care Program aims to reduce the progression and complications of chronic disease, improve the quality of life of people with chronic diseases and support their carers and families. It also aims to reduce unplanned and avoidable admissions to hospitals and improve the health systems capacity to respond to the needs of people with chronic diseases. Many Aboriginal people will be eligible, however issues around access, engagement and identification will 
present as barriers for enrolment to this program. The NSW Health Primary Health, Community Partnerships and Chronic Disease Branch has endorsed the targeting of the Connecting Care Program enhancement funds to provide positions dedicated to Aboriginal enrolments in each Local Health District. It is anticipated that joint planning will occur between the Connecting Care Program, Chronic Care for Aboriginal People program, the Division of General Practice and Aboriginal Medical Services to integrate positions, programs and service provision.

\section{Conclusion}

The Chronic Care for Aboriginal People program, the culmination of many years of work, will continue to evolve to support flexible local approaches to chronic disease. The implementation of the 48 Hour Follow-up program across NSW has been a success of the program. Aboriginal people should feel safe and confident to access all health services and have choice in their own health care. To allow choice it is therefore important to maintain Aboriginal-specific services as well as ensuring mainstream services are better able to meet their needs.

\section{References}

1. Australian Institute of Health and Welfare. Australia's health 2010. Australia's health no. 12. Cat. no. AUS 122. Canberra: AIHW, 2010.

2. Centre for Epidemiology and Research. Health of the people of New South Wales - Report of the Chief Health Officer, 2010.
Sydney: NSW Department of Health, 2010. Summary Report Available at: http://www.health.nsw.gov.au/pubs/2010/pdf/ chorep_summary_2010.pdf (Cited 2 January 2012).

3. Australian Bureau of Statistics. The Health and Welfare of Australia's Aboriginal and Torres Strait Islander Peoples. Oct 2010. Updated 14/04/2011. 4704.0 Available at: http://www. abs.gov.au/AUSSTATS/abs@.nsf/lookup/4704.0Main+ Features1Oct+2010 (Cited 2 February 2012).

4. NSW Health. Aboriginal Chronic Conditions Area Health Service Standards. Cardiovascular disease, diabetes, kidney disease, chronic respiratory disease and cancer. 2005. Available at: Aboriginal Chronic Conditions Area Health Service Standards (Cited 24 April 2012).

5. Ben-Tovim DI, Dougherty ML, O'Connell TJ, McGrath KM Patient journeys: the process of clinical redesign. Med J Aust 2008; 188(6): S14-7.

6. Health NSW. The Walgan Tilly Project: Chronic Care for Aboriginal People Final Report. April 2008. Available at: http://www.archi.net.au/_data/assets/pdf_file/0008/58247/ walgantilly.full.pdf (Cited 2 January 2012).

7. NSW Health. Clinical services redesign program. Chronic care for Aboriginal people. September 2010. Available at: http:// www.health.nsw.gov.au/resources/Initiatives/chronic_care/ aboriginal/pdf/CCAP_MoC_December2010.pdf (Cited 2 January 2012).

8. Council of Australian Governments. National Partnership Agreement on Closing the Gap in Indigenous Health Outcomes: an agreement between the Commonwealth of Australia and the States and Territories. 1 July 2009. Available at: http://www. coag.gov.au/intergov_agreements/federal_financial_relations/ docs/national_partnership/NP_closing_the_Gap_indigenous_ health_outcomes.pdf (Cited 24 April 2012). 